\title{
Characterisation of NeISSERIA meningitidis C Strains CAUSING TWO CLUSTERS IN THE NORTH OF ITALY IN 2007 AND 2008
}

\author{
C Fazio ${ }^{1}$, A Neri ${ }^{1}$, S Tonino ${ }^{1}$, A Carannante ${ }^{1}$, M G Caporali², S Salmaso², P Mastrantonio (paola.mastrantonio@iss.it) ${ }^{1}$, P \\ Stefanelli ${ }^{1}$ \\ 1. Department of Infectious, Parasitic and Immune-mediated Diseases, Istituto Superiore di Sanità, Rome, Italy \\ 2. National Centre for Epidemiology, Surveillance and Health Promotion, Istituto Superiore di Sanità, Rome, Italy
}

\begin{abstract}
Two clusters of invasive meningococcal disease in the north of Italy both due to serogroup C/ST-11 clonal complex are here described. The objective of the investigation was to analyse the phenotype and the genotype of meningococci involved in the two clusters which were of national relevance due to the fatal outcome of the majority of cases (six of the total of 10 cases). All the strains were C:2a:P1.5 ST-11/ET-37 clonal complex. Two pulsed field gel electrophoresis (PFGE) and variable number tandem repeats (VNTR) profiles were identified, one for each cluster. VNTRs were different from those detected in Italy for C/ST-11 strains isolated from sporadic cases in the same period.

This laboratory surveillance report highlights the importance and the crucial role of molecular characterisation to confirm the relatedness among meningococci responsible for clusters of cases.
\end{abstract}

\section{Introduction}

Meningococcal disease remains a major childhood infection in Europe, with a considerable number of cases appearing also in other age groups, notably young adults. The incidence of serogroup $C$ disease substantially declined with the introduction of conjugate meningococcal $C$ vaccine in the national vaccination programmes of several countries [1]. However, the C/ST-8 and the C/ST-11 strains are currently the two hyper-virulent meningococcal lineages involved in a significant proportion of serogroup $C$ invasive disease worldwide [2].

In Italy, the notification of invasive meningococcal disease to the local health authorities and to the Ministry of Health has been mandatory since 1983. Through national surveillance of bacterial meningitis, established in 1994, the National Reference Laboratory (NRL) at the Istituto Superiore di Sanità in Rome each year receives an average of $80 \%$ of strains isolated by local hospital laboratories throughout the country. The disease, characterised by low national incidence $(0.3 / 100,000$ inhabitants) and by sporadic cases, has in the last three years mainly been caused by serogroup B meningococci (64\%).

Since the end of 2007, two clusters of serogroup C meningococcal disease have been detected in two different administrative regions in Italy. Due to the severity and fatal outcome of cases, these clusters were of national relevance, and the strains have been fully characterised at the NRL. The molecular characteristics of the ten strains involved in the two clusters are reported here.

\section{Methods}

Isolates of meningococci received at the NRL were subcultured for serogroup confirmation by slide agglutination with commercial antiserum (Remel Europe, United Kingdom). Serotypes and serosubtypes were determined by standard whole-cell ELISA with monoclonal antibodies (purchased from NIBSC, UK) [3]. Susceptibilities to penicillin G, rifampicin, ciprofloxacin and ceftriaxone were determined by E-test method (AB Biodisk, Solna, Sweden), according to the manufacturer's instructions.

The breakpoints were those recommended by the European Monitoring Group for Meningococci EMGM [4].

Molecular analyses by multilocus sequence typing (MLST), variable number of tandem repeats (VNTR) typing and pulsed field gel electrophoresis (PFGE) were performed following the procedures described elsewhere [5-7].

\section{Results}

The two clusters of serogroup $\mathrm{C}$ meningococci occurred in a group of seven adolescents/young adults and in three adults in two different but bordering Italian regions, Veneto and Lombardy, in December 2007 and in July 2008, respectively.

The outbreak in Veneto has already been described from an epidemiological aspect and in terms of management [8]. The outcome was fatal in three of the seven cases.

From 13 to 15 July 2008, three cases of fatal septicaemia in patients aged 34, 48 and 51 years occurred in a limited geographical area of the Lombardy region. Family members and people who had been in contact with the patients were given chemoprophylaxis. Thorough investigation by the local health authorities did not show any social or institutional link between the three cases, and none could be identified as specifically at risk on the basis of the information obtained. All cases from both events, were laboratory-confirmed at the regional level by culture and the serogroup of Neisseria meningitidis was identified. At the NRL, the phenotypic and genotypic characteristics of the ten isolates were further determined. The strains showed the antigenic formula C:2a:P1.5 and were fully susceptible to penicillin, rifampicin, ceftriaxone and ciprofloxacin. All of them belonged to ST-11/ET37 clonal complex (cpx) as identified by MLST. 
PFGE confirmed the relatedness of strains within each cluster (Figure, Panel A, lanes 3-9 and 11-13). In particular, the presence of a single pattern from each cluster was observed.

VNTR analysis was also performed to further discriminate among ST-11 strains. The isolates showed a high degree of similarity in the patterns identified for each cluster and were different compared to VNTR profiles found among C/ST-11 strains isolated sporadically in the country in the same period (Figure, Panel B, lanes 3-9 and 11-13).

\section{Discussion}

Vaccination campaigns in Europe [1,9] against $N$. meningitidis serogroup $C$ have been very effective and have contributed significantly to its decline mainly among children and adolescents. However, the spread of ST-11 hyper-virulent meningococci among non-vaccinees is noteworthy due to the high transmissibility and low carriage rate, as documented by the literature $[1,10]$. A thorough assessment based on clinical and laboratory diagnosis combined with genotyping of all strains isolated during a cluster is highly recommended to confirm the clonality and to detect the circulation of new variants in this hypervirulent complex [1].

In this report, two clusters caused by $\mathrm{C}: 2 \mathrm{a}: \mathrm{P} 1.5 / \mathrm{ST}-11$ meningococci in December 2007 and July 2008 in northern Italy, have been reported. The two events are of national relevance due to

\section{F I G U R E}

Genetic relationship among meningococci C:2a:P1.5/ST-11 by PFGE (panel A) and VNTR analysis (panel B). Molecular analyses of isolates from clusters of Neisseria meningitidis infection in

Veneto and Lombardy, Italy, between December 2007 and July 2008

A
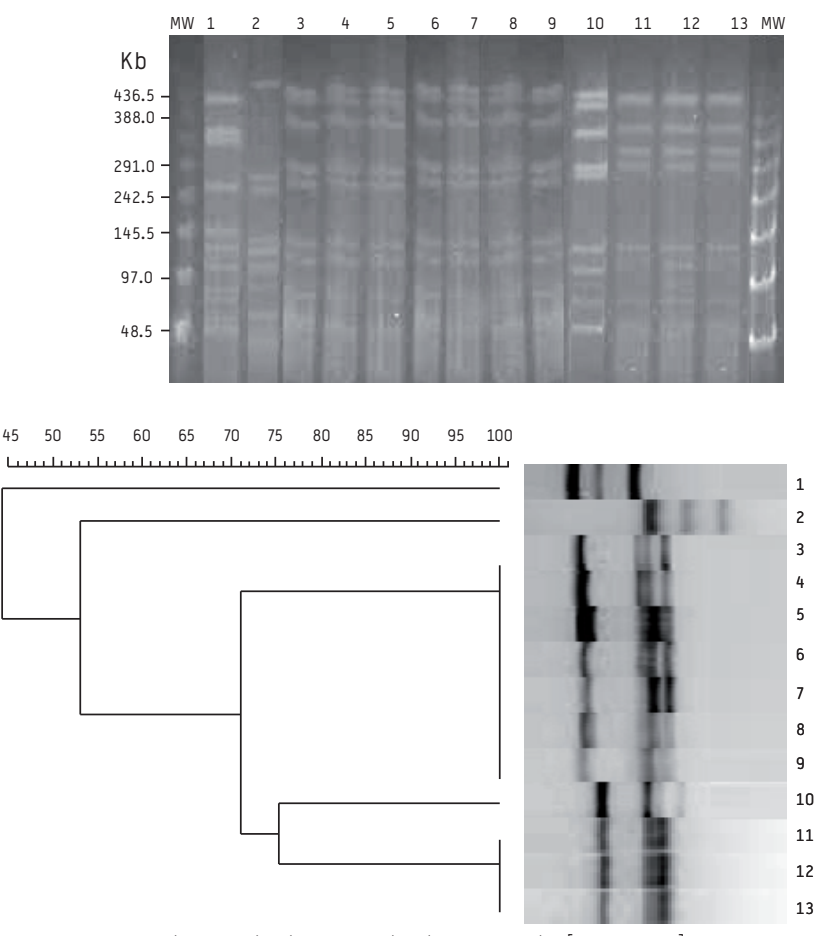

DICE (0pt: 1.00\%) (Tol 1.0\%-1.0\%) $\quad(H>0.0 \% \mathrm{~S}>0.0 \%) \quad[0.0 \%-100.0 \%]$

Lanes 1-2 and 10, C:2a:P1.5/ST11 meningococci isolated sporadically in Italy; lanes 3-9 and 11-13, C:2a:P1.5/ST-11 from clusters in Veneto and Lombardy, respectively. MW: molecular weights (New England, Biolabs). The left side of the VNTR gel photo is the top of the gel. VNTR: Variable number tandem repeat the high fatality rate of the disease. The molecular studies (PFGE and VNTR) performed at the NRL demonstrated the involvement of two different clones, each responsible for a cluster. Interestingly, VNTR analysis identified profiles not yet detected among other C:2a:P1.5/ST-11 strains circulating in the country over the last few years.

The present analysis confirms that, from a public health perspective, genotyping in the investigation of a cluster is crucial to detect the circulation of a hyper-virulent clone, to identify new variants and to monitor the spread in the area.

\section{Aknowledgements}

The authors thank Professor G Palù and Dr S Parisi of Veneto Regional Reference Laboratory, Dr E Torresani and Dr ML Garlaschi of Lombardy Regional Reference Laboratory and the microbiologists of the hospital laboratories participating in the Italian National Surveillance of Invasive Bacterial Diseases.

The authors wish to acknowledge the work of Dr AM Dionisi for the VNTR data analysis by using BioNumerics software.

This study made use of the Neisseria MultiLocus Sequence Typing website (http://neisseria.mlst.net) developed by $\mathrm{Dr}$ M-S Chan and sited at the University of Oxford. The development of this site is funded by the Wellcome Trust.

This work was partially funded by the Ministry of Health-CCM Project 116 "Surveillance of invasive bacterial diseases" 2007-2009.

\section{References}

1. Alonso JM, Gilmet G, Rouzic EM, Nassif X, Plotkin SA, Ramsay M, et al. Workshop on vaccine pressure and Neisseria meningitidis, Annecy, France, 9-11 March 2005. Vaccine. 2007;25(21):4125-9.

2. Lancellotti M, Guiyoule A, Ruckly C, Hong E, Alonso JM, Taha MK. Conserved virulence of $C$ to $B$ capsule switched Neisseria meningitidis clinical isolates belonging to ET-37/ST-11 clonal complex. Microbes Infect. 2006;8(1):191-6.

3. Abdillahi H, Poolman JT. Definition of meningococcal class 1 OMP subtyping antigens by monoclonal antibodies. FEMS Microbiol Immunol. 1988;1(3):139-44

4. Vazquez JA. Resistance testing of meningococci: the recommendations of the European Monitoring Group on Meningococci. FEMS Microbiol Rev. 2007;31(1):97100.

5. Maiden MC, Bygraves JA, Feil E, Morelli G, Russell JE, Urwin R, et al. Multilocus sequence typing: a portable approach to the identification of clones within populations of pathogenic microorganisms. Proc Natl Acad Sci U S A. 1998;95(6):3140-5.

6. Hartstein AI, Chetchotisakd P, Phelps CL, LeMonte AM. Typing of sequential bacterial isolates by pulsed-field gel electrophoresis. Diagn Microbiol Infect Dis. 1995;22(4):309-14

7. Yazdankhah SP, Lindstedt BA, Caugant DA. Use of variable-number tandem repeats to examine genetic diversity of Neisseria meningitidis. J Clin Microbiol. 2005;43(4):1699-705.

8. Ferro A, Baldo V, Cinquetti S, Corziali P, Gallo G, Lustro G, et al. Outbreak of serogroup $C$ meningococcal disease in Veneto region, Italy. Euro Surveill. 2008:13(2):pij=8008. Available from: http://www.eurosurveillance.org/ ViewArticle. aspx?ArticleId $=8008$

9. Trotter $\mathrm{CL}$, Ramsay ME. Vaccination against meningococcal disease in Europe: review and recommendations for the use of conjugate vaccines. FEMS Microbiol Rev. 2007;31(1):101-7.

10. Claus H, Maiden MC, Wilson DJ, McCarthy ND, Jolley KA, Urwin R, et al, Genetic analysis of meningococci carried by children and young adults. J Infect Dis. 2005;191(8):1263-71.

This article was published on 23 April 2009.

Citation style for this article: Fazio C, Neri A, Tonino S, Carannante A, Caporali MG, Salmaso S, Mastrantonio P, Stefanelli P. Characterisation of Neisseria meningitidis $C$ strains causing two clusters in the north of Italy in 2007 and 2008. Euro Surveill. 2009;14(16):pii=19179. Available online: http://www.eurosurveillance.org/ViewArticle. aspx?ArticleId $=19179$ 